\title{
Genetic recombination in plant-infecting messenger-sense RNA viruses: overview and research perspectives
}

\author{
Jozef J. Bujarski ${ }^{1,2 *}$ \\ Plant Molecular Biology Center and the Department of Biological Sciences, Northern Illinois University, DeKalb, IL, USA \\ 2 Laboratory of Molecular and Systems Biology, Institute of Bioorganic Chemistry, Polish Academy of Sciences, Poznan, Poland
}

\author{
Edited by: \\ Jean-François Laliberté, Institut \\ national de la recherche scientifique, \\ Canada \\ Reviewed by: \\ F. Murilo Zerbini, Universidade \\ Federal de Viçosa, Brazil \\ Justin Pita, Pennsylvania State \\ University, USA \\ *Correspondence. \\ Jozef J. Bujarski, Plant Molecular \\ Biology Center and the Department \\ of Biological Sciences, Northern \\ Illinois University, Montgomery Hall, \\ DeKalb, IL 60115, USA. \\ e-mail: jbujarski@niu.edu
}

RNA recombination is one of the driving forces of genetic variability in (+)-strand RNA viruses. Various types of RNA-RNA crossovers were described including crosses between the same or different viral RNAs or between viral and cellular RNAs. Likewise, a variety of molecular mechanisms are known to support RNA recombination, such as replicative events (based on internal or end-to-end replicase switchings) along with non-replicative joining among RNA fragments of viral and/or cellular origin. Such mechanisms as RNA decay or RNA interference are responsible for RNA fragmentation and trans-esterification reactions which are likely accountable for ligation of RNA fragments. Numerous host factors were found to affect the profiles of viral RNA recombinants and significant differences in recombination frequency were observed among various RNA viruses. Comparative analyses of viral sequences allowed for the development of evolutionary models in order to explain adaptive phenotypic changes and co-evolving sites. Many questions remain to be answered by forthcoming RNA recombination research. (1) How various factors modulate the ability of viral replicase to switch templates, (2) What is the intracellular location of RNA-RNA template switchings, (3) Mechanisms and factors responsible for non-replicative RNA recombination, (4) Mechanisms of integration of RNA viral sequences with cellular genomic DNA, and (5) What is the role of RNA splicing and ribozyme activity. From an evolutionary stand point, it is not known how RNA viruses parasitize new host species via recombination, nor is it obvious what the contribution of RNA recombination is among other RNA modification pathways. We do not understand why the frequency of RNA recombination varies so much among RNA viruses and the status of RNA recombination as a form of sex is not well documented.

Keywords: RNA recombination, viral replicase, template switching, non-replicative RNAs, host factors, cellular RNAs, ribonucleases, viral evolution

\section{INTRODUCTION}

Plus-stranded RNA viruses include some of the most dangerous pathogens for animals and humans. Moreover, a vast majority of plant viruses are $(+)$ RNA viruses. RNA viruses demonstrate a large level of variability in their genetic information, due to either mutations, RNA-RNA crossovers (RNA recombination), or reassortment. RNA recombination was demonstrated for many RNA virus species, whether under natural or experimental conditions. Similar to genetic recombination in DNA-based organisms, viral RNA recombination is defined as the process of swapping RNA fragments among RNA molecules. If crossovers occur amongst the same RNA templates in a homologous fashion, the exchanges are functionally equivalent to DNA meiotic crossing-over. In some viruses, the frequency of homologous crossing-over is very high and practically every replicated viral RNA molecule can be considered as chimerical in nature, as we have demonstrated for brome mosaic virus (BMV) RNAs (Urbanowicz et al., 2005).

A variety of events have been described that contribute to the formation of RNA recombinants (Figure 1). Such events include crossovers between viruses belonging to the same or to different taxonomic groups, between viruses infecting different hosts, or from adopting genetic material from the host. Numerous questions about molecular mechanisms of RNA recombination remain unanswered. This review attempts to summarize the most important venues of RNA recombination research, their challenges and future directions in order to draw more accurate models for this important RNA virus phenomenon. Since this issue of Frontiers concerns plant pathology, most of the material discusses RNA recombination in plant viruses. However, the less advanced aspects of plant recombination studies have been illustrated with examples taken from animal/human RNA viruses in order to show mutual possibilities for model research.

\section{REPLICATIVE MECHANISM OF RNA RECOMBINATION}

The generally accepted mechanism of RNA recombination is currently explained by a copy-choice model where the viral RNA polymerase (RdRp) complex in mRNA viruses [reverse transcriptase (RT) in retroviruses] changes templates during synthesis of the nascent strand (Galetto et al., 2006). This swapping process generates recombinant RNA molecules of mixed ancestry. Although we begin to understand the nature of these processes, many questions are waiting for an answer. One group 


\section{Mechanisms of Viral RNA recombination}

Internal template switching by viral $R d R p$

End-to-end template switching by viral RdRp

Primer-extension mechanisms

Replicase snatching at promoter regions
Role of template RNA sequence and structure

Role of RdRp and other viral proteins

Role of host factors

Role of promoters and binding sites

Role of RNA replication factories
Transesterification reactions

End-to-end template switching

Primer extension mechanisms

\section{Crossovers with nonreplicative RNAs}

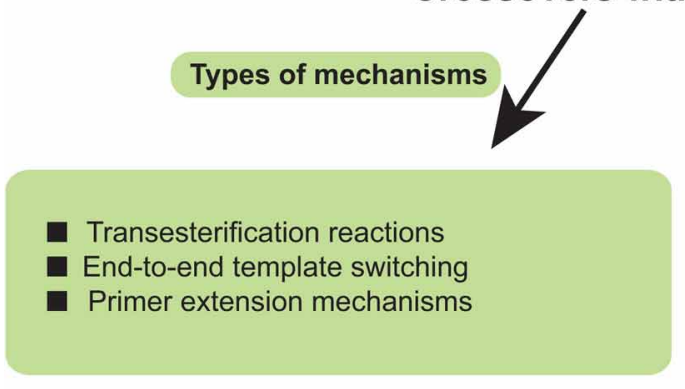

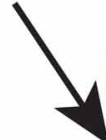

Role of cellular ribonucleases

Role of RNA decay pathways

- Role of RNA interference

- Role of RdRp and other viral proteins

- Role of template RNA sequence and structure

Role of RNA ligases?

Role of ribozymes?

Role of RNA splicing?

FIGURE 1 | Summary of types of mechanisms of genetic RNA recombination in (+) RNA viruses and factors influencing the RNA-RNA crossovers.

of questions revolves around the features that define the sites of crossovers. Among the factors known to promote replicase to switch are sequence homologies between recombination substrates along with secondary structures at the crossover sites, as demonstrated with the BMV and other systems (Figlerowicz and Bujarski, 1998; Nagy et al., 1999b). Also, the transcriptional activity seems to promote template switching. For instance, an efficient recombination hot spot has been mapped within the intercistronic region of BMV RNA3, the site carrying the promoter of transcription of subgenomic RNA4 (Wierzchoslawski et al., 2004). It is unknown what exactly facilitates crossovers at such sites. Possibilities include a snatching process of already bound RdRp complex to the promoter site, the premature termination of RNA synthesis and the replicase detachmentreattachment, or the effect of other bound viral and/or host factors (Sztuba-Solinska et al., 2011). These mechanisms may depend upon the type of template-switching process (whether the crosses occur internally or near the ends of the RNA templates) and on the involvement of crossover sequences in other processes, e.g., as a promoter of RNA replication or transcription.

Template switching was found to occur between related but also between unrelated RNA templates, generating legitimate (homologous) and illegitimate (nonhomologous) recombinants, respectively (Nagy and Simon, 1997). Since the latter involves sequences with little similarity, other factors must be important. Some data indicate that switches depend upon sequence composition, with the AU-rich regions promoting the RdRp detachment (Nagy et al., 1999a) and upon secondary structures (Galetto et al., 2006), along with protein or RNA binding activity. Switching may also depend upon the processivity (a measure of the average number of nucleotides copied per template association-disassociation cycle) features of the RdRp enzyme (Breyer and Matthews, 2001). A mandatory replicase breaking site is the end of any RNA template. End-to-end switching has been reported based upon in vitro results with RdRp enzymes from Bovine viral diarrhea virus (BVDV), BMV, Cucumber mosaic virus (CMV), and Cowpea chlorotic mottle virus (CCMV) (Kim and Kao, 2001). It is, however, not known how exactly such switches occur and whether the molecular mechanism is common among polymerases of different RNA viruses.

The strength of binding of the RdRp complex may play a key role during RNA template detachment-reattachment. With an increasing number of available RNA polymerase crystal structures, more is evident about the elements involved in RNAreplicase interactions. For instance, removal of a $\beta$-hairpin loop from the HCV RdRp protein increased de novo RNA synthesis and promoted RNA binding (Mosley et al., 2012). The RNA copying fidelity might be a matter of a nanosecond timescale complex dynamic in the RdRp enzyme that determines RNA 
binding, nucleotide binding or catalysis (Moustafa et al., 2011) and thus needs to be experimentally determined. The use of engineered replicase variants in RNA recombination assays will shed new light onto the molecular details of template switching mechanisms.

Another, not well answered question is how RNA template substrates come together in order to facilitate the switch. One possibility is that secondary structure regions can hybridize in trans bringing the two RNA templates into a local interaction. Such data are available, for instance, based upon limited observations in BMV (Nagy and Bujarski, 1993; Dzianott et al., 1995) or analogously, during switches between dimeric RNAs (within kissing loops) during reverse transcription inside the Human immunodeficiency virus Type-1 (HIV-1) virions (Nikolaitchik et al., 2011), an atypical $(+)$ sense RNA virus.

Yet, other data reveal that $(+)$ RNA viruses are replicating in membranous structures called spherules or replication factories (Laliberté and Sanfaçon, 2010). Such host membrane-derived replication vesicles have limited loading capacity, but they may carry up to several positive and negative strand RNA molecules (den Boon et al., 2010). Recent advances reveal the assembly of replicase complexes within replication factories via highly orchestrated interactions between viral proteins, viral genomic RNAs, and co-opted host factors (Mine and Okuno, 2012). Such a micro-environment may secure tight packaging and thus the closeness of internalized viral RNA molecules. From the formal stand point then, one may consider RNA recombination switches in $(+)$ RNA viruses inside replication factories as analogous to the switches that occur, e.g., during reverse transcription inside the HIV-1 virions.

Recently, we have demonstrated the participation of coat protein (CP) during BMV RNA recombination (Sztuba-Solinska et al., 2012). The nucleotide changes in cis-acting RNA motifs and the amino acid replacements within the corresponding $\mathrm{CP}$ binding sites-both debilitated the BMV RNA recombination. CP molecules likely mediate RNA crosses via dimerization/oligomerization of bound CP subunits. Indeed, the presence of BMV CP molecules has been demonstrated to be inside replication vesicles (Bamunusinghe et al., 2011). Another untested possibility predicts that a bound $\mathrm{CP}$ functions as a road block catalyzing the detachment of the replicase complex. The CP may also affect the properties of viral replicase. For instance, it has been shown recently that Norovirus RNA synthesis was enhanced by co-expressed structural protein VP1 (Subba-Reddy et al., 2012).

\section{RECOMBINATION WITH NON-REPLICATIVE RNAs}

Besides replicative copy-choice, the non-replicative mechanisms of viral RNA recombination have been described, mainly for animal/human RNA viruses, with almost no research focusing plant viruses. One of the best characterized non-replicative processes is demonstrated in the poliovirus where viable viruses were rescued in cells co-transfected with different pairs of viral RNA fragments (Gmyl et al., 1999). It is likely the recombinants may have resulted from transesterification reactions with the end structures similar to known ribozymes via intermediary formation of $2^{\prime}, 3^{\prime}$-cyclic phosphate. Indeed, in vitro data show that the transesterification reactions in the bacteriophage Qbeta RNA are guided by secondary structures that direct the attack of a $3^{\prime}$ hydroxyl onto the phosphodiester bonds (Chetverin et al., 1997). Later observations revealed enormous variability of the poliovirus genome and some variants may have been introduced by genetic errors due to non-replicative mechanisms (Agol, 2006). More recent results with partially-complementary RNA-oligonucleotides demonstrated the spontaneous formation of novel RNA molecules via $3^{\prime}, 5^{\prime}$-phosphodiester bonds (Lutay et al., 2007). These data show that viral RNA recombination can occur without participation of the RNA polymerase enzyme. The exact mechanisms of these non-replicative events are not completely understood and require further studies.

In contrast to poliovirus and other picornaviruses, bacteriophage Qbeta demonstrates low levels of recombination frequency. By using a cell-free system, Chetverin et al. (2005) have shown a high yield of primer-extension recombination with poliovirus replicase, but a low yield with Qbeta replicase. Thus, RNA recombination by poliovirus vs. Qbeta RdRps must be mechanistically different. Although both utilize transesterification reactions, the precise molecular bases for RNA swappings used by each of these enzymes are likely dissimilar reflecting different biochemical adaptations to the needs of individual viruses. It would be interesting to confirm experimentally the proposed transesterification models.

Among other examples of non-replicative recombination in mRNA viruses, the co-transfections of replicating and nonreplicating rubella virus (RUB) RNA transcripts containing nonoverlapping deletions did restore the infectious virus (Adams et al., 2003). Both, homologous and nonhomologous RNA recombinants emerged. The mechanism seemed to involve end-to-end replicase switching after initiation of minus-strand synthesis. However, the details of such mechanisms have not yet been confirmed. Another example of that sort involves recombination between BVDV and cellular RNAs, which can occur in the absence of viral replicase (see section "Recombination Between Viral and Cellular RNAs"). Analogous studies in the area of plant virology remain to be performed.

\section{ROLE OF HOST FACTORS DURING RNA RECOMBINATION}

An important subject of RNA recombination research is the role of host factors. While the involvement of viral RdRp proteins has been studied extensively, knowledge of the functions host components play is limited (Nagy and Pogany, 2011). One study was done with a model system of tomato bushy stunt virus (TBSV) that can recombine in yeast cells. The authors screened a yeast knockout library to identify over thirty different host genes suppressing or accelerating the TBSV RNA recombination (Serviene et al., 2005; Nagy, 2011). An interesting example is the gene PMR1 which encodes an ion pump (Pmrlp) controlling the Mn2+ concentration which may consequently affect the ability of TBSV replicase to change RNA binding/template switching events. Also stress signals, e.g., salt stress, affect viral recombination indirectly, by changing the concentration of recombination-essential proteins. Future studies are required to understand the interrelated network of cellular factors that define the final outcome of TBSV 
RNA recombinants, not only in model yeast cells, but also in natural TBSV plant hosts. Moreover, these studies are limited to only one specific TBSV RNA experimental recombination system, and it is unclear if other RNA recombination events within the TBSV RNA follow similar mechanistic pathways.

In the copy choice mechanism, recombinant RNAs are formed due to switching of viral replicase among RNA templates. The switching properties likely depend on the co-recruited host factors. In BMV, a variety of host factors were found to be employed by the replicase complex (Noueiry and Ahlquist, 2003). Many of these factors facilitate the complex assembly, but some regulate viral gene expression or recruitment of BMV RNAs to the membrane replication factories. Yet, other factors modify lipid composition of the endoplasmic reticulum membrane which activates the replication complex. Many of these factors can potentially affect the co-recruitment of RNA recombination substrates and/or BMV replicase switching properties during recombination. BMV RNA recombination was reported to occur in yeast cells (Garcia-Ruiz and Ahlquist, 2006), but a systematic identification of host factors participating in BMV RNA recombination remains to be done. It will be interesting to find out whether these factors parallel those in the above tombusvirus recombination system. This data will broaden our knowledge about host pathways enabling RNA viruses to recombine their genetic information. As such, it will contribute to predictions made on the stability of the RNA viral genome in various hosts.

The functions of recruited host proteins and host membranes in different $(+)$ RNA virus systems are now being progressively elucidated. Comparison among three plant RNA virus replication systems (TBSV, BMV, and dianthoviruses) reveals general patterns within the stepwise process of viral replicase complex assembly which requires concerted involvement of protein-protein, RNA-protein, and protein-lipid interactions (Mine and Okuno, 2012). However, each of these three plant virus systems recruits its own array of specific host factors. This suggests that each RNA virus has significantly unique ways of adapting to the cellular environment in order to assemble a functional RNA replication complex. This further suggests specific requirements are needed for RNA recombination in each individual RNA virus and therefore the recombination characteristics may significantly differ with each other among RNA viruses. Crystal structure studies help to reveal the complex and individual nature of viral replicases. Examples being the structure of Q $\{$ beta $\}$ phage polymerase, determined by Takeshita and Tomita (2010), or the analysis of the crystal structure of tomato mosaic virus helicase as a component of the viral replicase complex (Nishikiori et al., 2012).

\section{RECOMBINATION BETWEEN VIRAL AND CELLULAR RNAs}

RNA recombination events between viral and cellular RNAs have been observed for both plant and animal RNA viruses. One example is RNA recombination between the BVDV, a member of the pestivirus genus, and cellular RNA sequences. It occurs at the presence yet also in the absence of an active viral RdRp enzyme, implying that the mechanisms must be different from replicative template switching events (Becher and Tautz, 2011). The case of BVDV recombination has practical implications because the recombinant virus is lethal to its host. Normally, the virus is persistent, limiting the efficiency of RNA replication due to the dependency of a viral protease on limiting amounts of a cellular cofactor. In general, the uptake of a variety of cellular protein coding sequences at various positions in the pestiviral genomes has been reported, demonstrating that pestiviruses can gain access to the RNA pool of their hosts via RNA recombination. The example of BVDV shows us not only that recombination events with cellular RNAs cannot be excluded for other viruses, but also that the recombinant RNAs can be retro-transcribed and occasionally integrated into the host genome. The exact molecular mechanisms of the crossover events with cellular RNAs remain to be elucidated, as well as what factors target the crossover sites both to viral and to cellular RNAs.

Besides BVDV, HIV-1 is known to recombine effectively with host RNAs, e.g., with host tRNAs after introducing its strong secondary structure elements into the HIV RNA (Konstantinova et al., 2007). HIV-1 is capable of acquiring new genetic material, especially to the RT-encoding ORF (van der Hoek et al., 2005; Berkhout, 2011). Information about similar recombinant crosses with host RNAs in plant RNA viruses remains very limited, and their mechanisms are waiting to be elucidated.

One recombination process that was addressed with plant viruses has been the events between an invading virus and the transgene mRNAs in transgenic plants (Aaziz and Tepfer, 1999). One such example being recombination between two strains of CMV where one strain was expressed as a transgene while the other strain infected the transgenic plant (Turturo et al., 2008). This research group has also described recombination between related viruses (CMV and tomato aspermy virus TAV), with the population of recombinants being similar to each other in transgenic and in nontransgenic plants, suggesting similar molecular mechanisms of recombination (Jacquemond, 2012). In general, this demonstrates that transgene viral mRNAs enter the same pathway as do natural viral RNAs, most likely operating in the cytoplasm.

RNA recombination between viral and micro (mi)RNAs has not yet been reported. However, given the fact that this would be a useful source of already adapted elements to be acquired by the virus in order to secure the in-trans host-gene regulation, the lack of commonality of such an acquisition is surprising. Since $(+)$ RNA viruses operate in the cytoplasm, as the miRNAs do, there are likely either structural and/or functional constrains against such snatching events. Future studies will certainly bring further insight to this question.

Recently, a reverse scenario was observed. Nonretroviral RNA sequences of Bornaviruses and other $(-)$ strand RNA viruses were integrated into the host genome, including the human genome (Belyi et al., 2010; Horie et al., 2010). Also, mRNA viruses were described to leave their sequences in the cellular DNA of infected hosts (Crochu et al., 2004; Anne and Sela, 2005; Maori et al., 2007; Zemer et al., 2008; Geuking et al., 2009). These results demonstrate that RNA viruses can serve as a source of genetic innovation for their hosts. The RT activity encoded by retrotransposons is most likely responsible for reverse transcription and integration, yet further molecular studies are needed.

The above examples illustrate that the cytoplasmic RNA processing mechanisms are able to cross paths with viral replication 
pathways inside the cell. Despite diverse examples of viral RNAs recombining with host RNA sequences (and vice versa), many unanswered questions remain to be addressed. They include, but are not limited to, the sub-cellular location of recombination events, the role and availability of host RNA degradome for recombination, or the link between the elements of RNA degradation pathways and viral RNA recombination. The molecular mechanisms of such crossover events are not well understood, especially whether template-switching or re-ligation processes are involved. More data, especially from plant RNA virus systems are required to assess the general nature of these processes in plant vs. animal/human tissues.

\section{ROLE OF RIBONUCLEASES AND RNA INTERFERENCE PATHWAYS}

Host RNAs undergo extensive degradation and turnover, as do viral RNAs (Lloyd, 2012). The participation of RNA decay pathways in viral RNA recombination has been studied in TBSV by the Nagy group (Jiang et al., 2010; Jaag et al., 2011). By testing eight known endoribonucleases, the authors have shown that mutations in the components of RNase MRP debilitated the production of endoribonucleolytically cleaved TBSV RNA in yeast. Also, by knocking down the RNase NME1 or silencing the Xrn4p exoribonuclease in Nicotiana benthamiana, the production of cleaved TBSV RNAs was debilitated, but recombination increased, suggesting the role of RNA intermediates in recombination (Jaag and Nagy, 2009). Similar effects promoting RNA recombination were observed in yeast for Xrn1p exoribonuclease (Serviene et al., 2005). It is noteworthy that deletions of the host Met22p/Hal2p bisphosphate- $3^{\prime}$-nucleotidase (a known inhibitor of the Xmn1p ribonuclease) or the inhibition of this nucleotidase with $\mathrm{LiCl}$ or $\mathrm{NaCl}$, also increased the frequency of TBSV RNA recombination in yeast (Jaag and Nagy, 2010). This shows that besides host factors, the salt stress can also affect viral RNA recombination. Whether other environmental conditions can influence viral RNA recombination needs further studies.

In contrary to RNA decay enzymes, we observed debilitating effects of the host RNA interference gene knockouts on BMV RNA recombination in Arabidopsis thaliana, and that BMV RNA fragments have recombined with BMV RNA progeny (Dzianott et al., 2012). It appeared that RNA silencing (RNAi) pathways participated in the rearrangement of genomic BMV RNAs. Therefore, BMV RNAs can recombine via several mechanisms including template-switching events along with RNAibased sequence swapping. Similarly, the promoting role of RNAi in viral RNA recombination was reported for mycovirus infection in chestnut blight fungus cells (Sun et al., 2009; Nuss, 2011). These two examples show that the RNAi mechanisms can function as antiviral tools, but also that RNA silencing can promote additional variability to the viral RNA genome. Further studies are needed to determine the formation of viral RNA recombinants from RNAi-induced degradation products.

\section{THE PHYLOGENETIC AND EVOLUTIONARY ROLE OF RNA RECOMBINATION}

The biological diversity within both plant and animal RNA viruses is one of the largest found in all other forms of nature.
RNA recombination is a main contributor to the ever evolving RNA viral genome. Comparative analyses of RNA viral sequences allow for the development of evolutionary models that demonstrate the associated adaptive phenotypic changes along with detecting the co-evolving sites within viral genomes (Pond et al., 2012).

The wide imprints of RNA recombination were found within natural populations of plant viruses. RNA recombination seems to be particularly frequent among members of the family Potyviridae, the largest family of plant RNA viruses. Frequent recombinational footprints were detected within the ORFs of both their structural and nonstructural proteins (Bousalem et al., 2000; Visser and Bellstedt, 2009; Yamasaki et al., 2010). Phylogenetic surveys indicate not only intraspecies and intragenus, but also intergenous recombination crossover's footprints in Potyviridae (Desbiez and Lecoq, 2004; Valli et al., 2007), supporting their apparent modular evolution. Recombination with host RNA was also detected, likely via retrotransposable elements (Tanne and Sela, 2005) demonstrating that, like animal viruses, plant viruses can expand their coding capacity via recombination with the host's messenger RNA pool (Chare and Holmes, 2006).

Also, the populations of plant viruses with genomes producing sgRNAs, e.g., Closteroviridae, Luteoviridae, or viruses with multipartite genomes, e.g., Bromoviridae, seem to accumulate recombinants readily. Evolutionary pathways were proposed for the emergence of members of Luteoviridae (Domier et al., 2002 and Moreno et al., 2004). Luteoviruses have mastered the process of modular swap (Pagán and Holmes, 2010) and the reconstructed phylogeny reveals their sequence evolution by intrafamilial as well as extrafamilial RNA recombination (Moonan et al., 2000). The most frequent swaps map to the junction between the CP and RdRp ORFs (Silva et al., 2008). In addition, some luteoviruses were found to recombine with host (chloroplast) sequences (Mayo and Jolly, 1991).

One extreme example of interspecies recombination is in circoviruses that arose by recombinants between plant DNA nanoviruses and mammalian RNA caliciviruses. In this case, the likely mediator has been a retrovirus that retro-transcribed the RNA into DNA (Davidson and Silva, 2008). Although likely, such events have not been experimentally confirmed and further research is required.

Among animal viruses, coronaviruses are highly recombinogenic (Woo et al., 2009) and natural RNA recombinant variants were described for flaviviruses (González-Candelas et al., 2011). By having one of the highest recombination rates among all viruses, retroviruses generate polymorphic sequences that increase their chances for survival under changing selection pressures (Delviks-Frankenberry et al., 2011). Besides retroviruses, picornaviruses are naturally highly recombinogenic (Lukashev, 2010). In fact, RNA recombination is their key genetic feature maintaining the global pool of variants from which the recombination snapshots generate new recombinant forms of picornaviruses. For instance, a model describing recombination between poliovirus and coxsackie virus was presented to illustrate the effects on viral emergence and evolution (Combelas et al., 2011). This and other studies reveal multiple mechanisms leading to genetic variability of polioviruses (Savolainen-Kopra 
and Blomqvist, 2010), with significant contribution of homologous recombination events that fix advantageous mutations or remove deleterious ones. However, further research is required to understand the detailed evolution mechanisms of polioviruses.

The evolutionary genetics of emerging plant RNA viruses was studied by Elena et al. (2011). Apparently, devastating virus epidemics can spread from new plant virus variants that acquired new virulence factors. This study shows a multifaceted picture of virus emergence. Changes in ecological conditions bring together the reservoir viruses and their crop hosts, often as a result of interplay among the environment, genetic plasticity, and the required host factors. The stochastic processes contribute to the beginning of viral emergence in a new host species, followed by the adaptation phase. Also, vectors impose strong bottlenecks during host-to-host transmissions. The reservoir population seems to be the most important determinant of viral emergence, but little is known about viruses of wild plant species that work as reservoirs.

For all the mentioned RNA virus systems, of either plants or animals, detailed roles during virus evolution of RNA secondary structures, the function of sequence similarity or the impact of RNA co-packaging during RNA recombination, are all not well understood. Inaccuracies of viral RNA replication, damage from environmental factors, and attacks by RNA-modifying enzymes, all can contribute to RNA genome corruption and thus generate a question of how RNA viruses maintain their genetic integrity (Barr and Fearns, 2010). It seems that viral RdRps are sufficiently flexible to accommodate alternative initiation mechanisms, enabling terminal repair, terminal transferase activity, and recombinational crosses in the case of damaged key terminal sequences. Among a variety of mechanisms to protect RNA viral genome integrity, recombination allows for exchange of sequences between RNA templates, protecting not only their entire genome, but also their vulnerable termini. A typical example of efficient terminal crossover exchanges is seen within the $3^{\prime}$ noncoding region of BMV RNAs (Bujarski and Kaesberg, 1986). The differences in replicase architecture might affect the predilection of a particular virus for RNA recombination. The molecular aspects of the theory on "adaptable" viral RdRps have not been elucidated and structural studies will contribute to the answers.

\section{METHODS FOR THE DETECTION OF RNA RECOMBINANTS}

RNA recombination research concerns both virus evolution (where the most important subject is the detection of recombination imprints among natural viral RNA sequences) and the mechanism of recombination (by using the experimental systems of enhanced recombination frequency). As regards to the evolutionary studies, various computer programs are used for massive comparisons of viral sequences in order to reveal the recombination footprints. The examples of such software include, but are not limited to, Topali, RECCO, GARD, RDP, GENECONV, Chimaera, MaxChi, BOOTSCAN, SISCAN, PHYLPRO, DIPLOMO, SImPlot, Lard, and 3SEQ. These programs can identify the recombination sites among different viral strains, different viral species, and even between the virus and the host (Chare and Holmes, 2006). With advent of next-generation massive sequencing, the genetic diversity of viral RNA genomes can be characterized through the mapping of polymorphisms and measurement of mutation frequencies as well as by detection of recombination events to a single-nucleotide resolution (Routh et al., 2012). Such approach is very sensitive and unbiased, and it can identify hundreds of thousands of recombination events, allowing for a detailed description of RNA crossover profiles.

The detection of recombination events in the laboratory is challenging because RNA-RNA crossovers apparently are rare events and thus the main effort is to elaborate on experimental systems of engineered RNA templates of increased recombination activity. The efficient recovery of recombinants in mixed infections could be achieved by using temperature sensitive mutants, a long-term method used for animal RNA viruses (Hirst, 1962; Pringle, 1970). In whole plants, an important problem is that most recombinants are not competitive with the parental types and therefore disappear. One way to increase recombination rate is by using viral mutants bearing sequence modifications at their UTRs, which decreases the replication abilities of parental molecules, as was used to detect the BMV recombinants (Bujarski and Kaesberg, 1986). Another approach utilizes viral RNAs bearing silent markers or via mixed infections with two low-competing viral strains (e.g., as the used by us mixed infection with both type and Fescue strains of BMV). Other plant virus recombination systems employ mixtures of two parental RNAs with one component carrying a deleterious mutation, e.g., satellite and genomic RNAs of TCV (Zhang et al., 1991), or defective interfering and genomic RNAs of TBSV, Cucumber necrosis virus (CNV) (White and Morris, 1995), and Potato virus X (PVX) (Draghici and Varrelmann, 2010). All these types of recombination systems can be used in cell-free extracts (utilizing viral RdRp preparations), in single-cell (protoplast) hosts, in whole plant hosts, and even in yeast. Some of the systems make use of transient expression vectors by agro-infiltrating plant leaves (Kwon and Rao, 2012).

With these systems in hand, virologists can address such aspects of the RNA recombination process as the essential role of RNA sequence and structure, especially the role of RNA motifs, the function of viral replicase (RdRp) and other viraland/or host-encoded proteins, or the mutual host-virus effects in short-term virus evolution. The main analytical effort in the recombination experiments is to identify RNA recombination products and to map the location of cross sites. Usually, viral RNAs are extracted and amplified by RT-PCR and the resulting cDNA products are cloned followed by sequencing and/or restriction digestion of a large number of clones. This way the crossovers are detected and mapped within the sequence markers, providing information about both frequency and distribution of recombination events. Proper controls are required to guard against RT-PCR generated recombinants.

\section{FINAL REMARKS: UNANSWERED QUESTIONS AND PERSPECTIVES}

Genetic RNA recombination is a major driving force for RNA virus diversity. By understanding the factors and the mechanisms that affect recombination, one can ultimately develop better means for controlling RNA virus infections. In this review I have described the current status of RNA virus recombination research and its future directions. I have also noted its progress over the 
last several years emphasizing on some future research venues. Evidently, there is still much to be learned about the mechanistic details of RNA recombination. For example, it is not yet clear how various factors modulate the ability of viral replicase to switch templates, such as the role of RNA template structures, the molecular and structural features of replicase proteins, or the functions of other viral and host factors during cross-over events. Also, the intracellular location of the RNA-RNA template switching has not been confirmed. Besides copy-choice, RNA viruses can recombine with non-replicative RNAs. It is not exactly known what mechanism is responsible for ligation of viral RNA fragments, or where inside the cell this process occurs. RNA viruses were found to recombine with cellular RNAs, but again where in the cell and what factors enable such events, is not well known. And the opposite, the exact steps and the molecular mechanisms of the RNA viral sequence integration with the cellular DNA have not been untangled. Amongst other questions, not much is known about how splicing or active ribozymes can contribute to the RNA virus recombination (Edgell et al., 2011).

From the evolutionary stand point, RNA recombination seems to play a key function during virus speciation and emergence, but its shared contribution that parallels other RNA modification pathways has not yet been assessed. We do not fully understand how RNA viruses achieve their high potential of parasitizing new host species via recombination (Domingo, 2010). The entire population of RNA variants that are present in reservoir hosts can now be determined with the tools of next-generation sequencing so that the role of recombinants can be more precisely evaluated (Beerenwinkel et al., 2012).

The frequency of RNA crossing-over varies among RNA virus species and there is little evidence that recombination was

\section{REFERENCES}

Aaziz, R., and Tepfer, M. (1999). Recombination in RNA viruses and in virus-resistant transgenic plants. J. Gen. Virol. 80, 1339-1346.

Adams, S. D., Tzeng, W. P., Chen, M. H., and Frey, T. K. (2003). Analysis of intermolecular RNA-RNA recombination by rubella virus. Virology 309, 258-271.

Agol, V. I. (2006). Molecular mechanisms of poliovirus variation and evolution. Curr. Top. Microbiol. Immunol. 299, 211-259.

Anne, E., and Sela, I. (2005). Occurrence of a DNA sequence of a non-retro RNA virus in a host plant genome and its expression: evidence for recombination between viral and host RNAs. Virology 332, 614-622.

Bamunusinghe, D., Seo, J. K., and Rao, A. L. (2011). Subcellular localization and rearrangement of endoplasmic reticulum by Brome mosaic virus capsid protein. J. Virol. 85, 2953-2963.

Barr, J. N., and Fearns, R. (2010). How RNA viruses maintain their genome integrity. J. Gen. Virol. 91, 1373-1387.

Becher, P., and Tautz, N. (2011). RNA recombination in pestiviruses: cellular RNA sequences in viral genomes highlight the role of host factors for viral persistence and lethal disease. RNA Biol. 8, 216-224.

Beerenwinkel, N., Günthard, H. F., VolkerRoth, and Metzner, K. J. (2012). Challenges and opportunities in estimating viral genetic diversity from next-generation sequencing data. Front. Microbiol. 3:329. doi: 10.3389/fmicb.2012.00329

Belyi, V. A., Levine, A. J., and Skalka, A. M. (2010). Unexpected inheritance: multiple integrations of ancient bornavirus and ebolavirus/marburgvirus sequences in vertebrate genomes. PLoS Pathog. 6:e1001030. doi: 10.1371/journal.ppat.1001030

Berkhout, B. (2011). HIV-1 as RNA evolution machine. RNA Biol. 8, 225-229.

Bousalem, M., Douzery, E. J. P., and Fargette, D. (2000). High genetic diversity, distant phylogenetic

favored by natural selection. Because of this and since recombination rates follow the patterns of RNA genome organization, Simon-Loriere and Holmes (2011) postulate that RNA recombination is a by-product of viral genome arrangement acting on selected aspects of the virus life cycle. Thus, according to the authors, RNA recombination does not seem to function as an obligatory form of sex in RNA viruses. Yet further studies are required, especially since Muller's ratchet effects were observed in RNA viruses (Turner, 2003) and the chimeric nature of viral RNAs due to frequent homologous RNA swaps was determined, e.g., in BMV (Urbanowicz et al., 2005).

Despite the above deficiencies, the so far accumulated knowledge about viral RNA recombination has already found some practical applications. For example, measures could be taken to reduce recombination while designing the antiviral resistance in transgenic plants with artificial micro RNAs (Fahim and Larkin, 2013) or with double stranded RNA-expressing transgenes (Zhang et al., 2011). Also, the potential instability and recovery of the wild-type virus via recombination can be reduced during construction of plant RNA viral vectors (Nagyová and Subr, 2007).

\section{ACKNOWLEDGMENTS}

The author thanks Margaret Bujarska for valuable comments on this manuscript. Jozef J. Bujarski was supported through a grant from the National Science Foundation (MCB-0920617) and through the Plant Molecular Biology Center at Northern Illinois University. The author apologizes to any author that has been omitted in this review for either space reasons or due to the nature of composition of this article.

relationships and intraspecies recombination events among natural populations of Yam mosaic virus: a contribution to understanding potyvirus evolution. J. Gen. Virol. 81, 243-255.

Breyer, W. A., and Matthews, B. W. (2001). A structural basis for processivity. Protein Sci. 10, 1699-1711.

Bujarski, J. J., and Kaesberg, P. (1986). Genetic recombination between RNA components of a multipartite plant virus. Nature 321, 528-531.

Chare, E. R., and Holmes, E. C. (2006). A phylogenetic survey of recombination frequency in plant RNA viruses. Arch. Virol. 151, 933-946.

Chetverin, A. B., Chetverina, H. V., Demidenko, A. A., and Ugarov, V. I. (1997). Nonhomologous RNA recombination in a cell-free system: evidence for a transesterificationmechanism guided by secondary structure. Cell 88, 503-513.

Chetverin, A. B., Kopein, D. S., Chetverina, H. V., Demidenko, A. A., and Ugarov, V. I. (2005). Viral RNA-directed RNA polymerases use diverse mechanisms to promote recombination between RNA molecules. J. Biol. Chem. 280, 8748-8755.

Combelas, N., Holmblat, B., Joffret, M. L., Colbère-Garapin, F., and Delpeyroux, F. (2011). Recombination between poliovirus and coxsackie A viruses of species C: a model of viral genetic plasticity and emergence. Viruses 3, 1460-1484.

Crochu, S., Cook, S., Attoui, H., Charrel, R. N., De Chesse, R., Belhouchet, M., et al. (2004). Sequences of flavivirus-related RNA viruses persist in DNA form integrated in the genome of Aedes spp. mosquitoes. J. Gen. Virol. 85, 1971-1980.

Davidson, I., and Silva, R. F. (2008). Creation of diversity in the animal virus world by inter-species and intra-species recombinations: lessons learned from poultry viruses. Virus Genes 36, 1-9.

Delviks-Frankenberry, K., Galli, A., Nikolaitchik, O., Mens, H., Pathak, V. K., and Hu, W. S. (2011). Mechanisms and factors that 
influence high frequency retroviral recombination. Viruses 3, 1650-1680.

den Boon, J. A., Diaz, A., and Ahlquist, P. (2010). Cytoplasmic viral replication complexes. Cell Host Microbe. 8, 77-85.

Desbiez, C., and Lecoq, H. (2004). The nucleotide sequence of watermelon mosaic virus (WMV, Potyvirus) reveals interspecific recombination between two related potyviruses in the 5_ part of the genome. Arch. Virol. 149, 1619-1632.

Domier, L. L., McCoppin, NK, Larsen, R. C., and D'Arcy, C. J. (2002). Nucleotide sequence shows that bean leafroll virus has a luteoviruslike genome organization. J. Gen. Virol. 83, 1791-1798.

Domingo, E. (2010). Mechanisms of viral emergence. Vet. Res. 41, 38-45.

Draghici, H.-K., and Varrelmann, M. (2010). Evidence for similarityassisted recombination and predicted stemloop structure determinant in potato virus X RNA recombination. J. Gen. Virol. 91, 552-562.

Dzianott, A., Flasinski, S., and Bujarski, J. J. (1995). Foreign complementary sequences facilitate genetic RNA recombination in brome mosaic virus. Virology 208, 370-375.

Dzianott, A., Sztuba-Solinska, J., and Bujarski, J. J. (2012). Mutations in the antiviral RNAi defense pathway modify Brome mosaic virus RNA recombinant profiles. Mol. Plant Microbe Interact. 25, 97-106.

Edgell, D. R., Chalamcharla, V. R., and Belfort, M. (2011). Learning to live together: mutualism between self-slicing introns and their hosts. BMC Biol. 9:22. doi: 10.1186/1741-7007-9-22

Elena, S. F., Bedhomme, S., Carrasco, P., Cuevas, J. M., de la Iglesia, F., Lafforgue, G., et al. (2011). The evolutionary genetics of emerging plant RNA viruses. Mol. Plant Microbe Interact. 24, 287-293.

Fahim, M., and Larkin, P. J. (2013). Designing effective amiRNA and multimeric amiRNA against plant viruses. Methods Mol. Biol. 942, 357-377.

Figlerowicz, M., and Bujarski, J. J. (1998). RNA recombination in brome mosaic virus, a model plus strand RNA virus. Acta Biochim. Pol. 45, 847-868.

Galetto, R., Giacomoni, V., Véron, M., and Negroni, M. (2006). Dissection of a circumscribed recombination hot spot in HIV-1 after a single infectious cycle. J. Biol. Chem. 281, 2711-2720.
Garcia-Ruiz, H., and Ahlquist, P. (2006). Inducible yeast system for Viral RNA recombination reveals requirement for an RNA replication signal on both parental RNAs. J. Virol. 80, 8316-8328.

Geuking, M. B., Weber, J., Dewannieux, M., Gorelik, E., Heidmann, T., Hengartner, H., et al. (2009). Recombination of retrotransposon and exogenous RNA virus results in nonretroviral cDNA integration. Science 323, 393-396.

Gmyl, A. P., Belousov, E. V., Maslova, S. V., Khitrina, E. V., Chetverin, A. B., and Agol, V. I. (1999). Non-replicative RNA recombination in poliovirus. J. Virol. 73, 8958-8965.

González-Candelas, F., López-Labrador, F., and Bracho, M. A. (2011). Recombination in hepatitis $\mathrm{C}$ virus. Viruses 3, 2006-2024.

Hirst, G. K. (1962). Genetic recombination with Newcastle disease virus, polioviruses, and influenza. Cold Spring Harb. Symp. Quant. Biol. 27, 303-309.

Horie, M., Honda, T., Suzuki, Y., Kobayashi, Y., Daito, T., Oshida, T., et al. (2010). Endogenous nonretroviral RNA virus elements in mammalian genomes. Nature 463, 84-87.

Jaag, H. M., Lu, Q., Schmitt, M. E., and Nagy, P. D. (2011). Role of RNase MRP in viral RNA degradation and RNA recombination. J. Virol. 85, 243-253.

Jaag, H. M., and Nagy, P. D. (2009). Silencing of Nicotiana benthamiana Xrn $4 p$ exoribonuclease promotes tombusvirus RNAaccumulation and recombination. Virology 386, 344-352.

Jaag, H. M., and Nagy, P. D. (2010). The combined effect of environmental and host factors on the emergence of viral RNA recombinants. PLoS Pathog. 6:e1001156. doi: 10.1371/journal.ppat.1001156

Jacquemond, M. (2012). Cucumber mosaic virus. Adv. Virus Res. 84, 439-504.

Jiang, Y., Cheng, C. P., Serviene, E., Shapka, N., and Nagy, P. D. (2010). Repair of lost $5^{\prime}$ terminal sequences in tombusviruses: rapid recovery of promoter- and enhancer-like sequences in recombinant RNAs. Virology 404, 96-105.

Kim, M. J., and Kao, C. (2001). Factors regulating template switch in vitro by viral RNA-dependent RNA polymerases: implications for RNARNA recombination. Proc. Natl. Acad. Sci. U.S.A. 98, 4972-4977.

Konstantinova, P., ter Brake, O., Haasnoot, J., de Haan,
P., and Berkhout, B. (2007). Trans-inhibition of HIV-1 by a long hairpin RNA expressed within the viral genome. Retrovirology 1, 4-15.

Kwon, S. J., and Rao, A. L. (2012) Emergence of distinct brome mosaic virus recombinants is determined by the polarity of the inoculum RNA. J. Virol. 86, 5204-5220.

Laliberté, J. F., and Sanfaçon, H. (2010). Cellular remodeling during plant virus infection. Annu. Rev. Phytopathol. 48, 69-91.

Lloyd, R. E. (2012). How do viruses interact with stress-associated RNA granules? PLoS Pathog. 8:e1002741. doi: 10.1371/journal.ppat.1002741

Lukashev, A. N. (2010). Recombination among picornaviruses. Rev. Med. Virol. 20, 327-337.

Lutay, A. V., Zenkova, M. A., and Vlassov, V. V. (2007). Nonenzymatic recombination of RNA: possible mechanism for the formation of novel sequences. Chem. Biodivers. 4 , 762-767.

Maori, E., Lavi, S., Mozes-Koch, R., Gantman, Y., Peretz, Y., Edelbaum, O., et al. (2007). Isolation and characterization of Israeli acute paralysis virus, a dicistrovirus affecting honeybees in Israel: evidence for diversity due to intra- and interspecies recombination. J. Gen. Virol. $88,3428-3438$.

Mayo, M. A., and Jolly, C. A. (1991). The $5^{\prime}$-terminal sequence of potato leafroll virus RNA: evidence of recombination between virus and host RNA. J. Gen. Virol. 72, 2591-2595.

Mine, A., and Okuno, T. (2012). Composition of plant virus RNA replicase complexes. Curr. Opin. Virol. 2, 663-669.

Moonan, F., Molina, J., and Mirkov, T. E. (2000). Sugarcane yellow leaf virus: an emerging virus that has evolved by recombination between luteoviral and poleroviral ancestors. Virology 269, 156-171.

Moreno, I. M., Malpica, J. M., D $\imath$ azPendo, J. A., Moriones, E., Fraile, A., and Garcia-Arenal, F. (2004) Variability and genetic structure of the population of watermelon mosaic virus infecting melon in Spain. Virology 318, 451-460.

Mosley, R. T., Edwards, T. E., Murakami, E., Lam, A. M., Grice, R. L., Du, J., et al. (2012). Structure of hepatitis $\mathrm{C}$ virus polymerase in complex with primer-template RNA. J. Virol. 86, 6503-6511.

Moustafa, I. M., Shen, H., Morton, B., Colina, C. M., and Cameron, C. E. (2011). Molecular dynamics simulations of viral RNA polymerases link conserved and correlated motions of functional elements to fidelity. J. Mol. Biol. 410, 159-181.

Nagy, P. D. (2011). The roles of host factors in tombusvirus RNA recombination. Adv. Virus Res. 81, 63-84.

Nagy, P. D., and Bujarski, J. J. (1993). Targeting the site of RNA-RNA recombination in brome mosaic virus with antisense sequences. Proc. Natl. Acad. Sci. U.S.A. 90, 6390-6394.

Nagy, P. D., Ogiela, C., and Bujarski, J. J. (1999a). Mapping sequences active in homologous RNA recombination in brome mosaic virus: prediction of recombination hot spots. Virology 254, 92-104.

Nagy, P. D., Pogany, J., and Simon, A. E. (1999b). RNA elements required for RNA recombination function as replication enhancers in vitro and in vivo in a plus-strand RNA virus. EMBO J. 18, 5653-5665.

Nagy, P. D., and Pogany, J. (2011). The dependence of viral RNA replication on co-opted host factors. Nat. Rev. Microbiol. 10, 137-149.

Nagy, P. D., and Simon, A. E. (1997). New insights into the mechanisms of RNA recombination. Virology 235, 1-9.

Nagyová, A., and Subr, Z. (2007). Infectious full-length clones of plant viruses and their use for construction of viral vectors. Acta Virol. 51, 223-237.

Nikolaitchik, O. A., Galli, A., Moore, M. D., Pathak, V. K., and $\mathrm{Hu}$, W.-S. (2011). Multiple Barriers to Recombination between Divergent HIV-1 Variants Revealed by a DualMarker recombination assay. J. Mol. Biol. 407, 521-531.

Nishikiori, M., Sugiyama, S., Xiang, H., Niiyama, M., Ishibashi, K., Inoue, T., et al. (2012). Crystal structure of the superfamily 1 helicase from Tomato mosaic virus. J. Virol. 86, 7565-7576.

Noueiry, A. O., and Ahlquist, P. (2003). Brome mosaic virus RNA replication: revealing the role of the host in RNA virus replication. Annu. Rev. Phytopathol. 41, 77-98.

Nuss, D. L. (2011). Mycoviruses, RNA silencing, and viral RNA recombination. Adv. Virus Res. 80, 25-48.

Pagán, I., and Holmes, E. C. (2010). Long-term evolution of the Luteoviridae: time scale and mode of virus speciation. J. Virol. 84, 6177-6187.

Pond, S. L., Murrell, B., and Poon, A. F. (2012). Evolution of viral genomes: interplay between selection, recombination, and other forces. Methods Mol. Biol. 856, 239-272.

Pringle, C. R. (1970). Genetic characteristics of conditional lethal 
mutants of vesicular stomatitis virus induced by 5 -fluorouracil, 5-azacytidine and ethyl methane sulfonate. J. Virol. 5, 559-567.

Routh, A., Ordoukhanian, P., and Johnson, J. E. (2012). Nucleotideresolution profiling of RNA recombination in the encapsidated genome of a eukaryotic RNA virus by next-generation sequencing. J. Mol. Biol. 424, 257-269.

Savolainen-Kopra, C., and Blomqvist, S. (2010). Mechanisms of genetic variation in polioviruses. Rev. Med. Virol. 20, 358-371.

Serviene, E., Shapka, N., Cheng, C. P., Panavas, T., Phuangrat, B., Baker, J., et al. (2005). Genomewide screen identifies host genes affecting viral RNA recombination. Proc. Natl. Acad. Sci. U.S.A. 102, 10545-10550.

Silva, T. F., Corrêa, R. L., Castilho, Y., Silvie, P., Bélot, J. L., and Vaslin, M. F. (2008). Widespread distribution and a new recombinant species of Brazilian virus associated with cotton blue disease. Virol. J. 5:123. doi: 10.1186/1743-422X-5-123

Simon-Loriere, E., and Holmes, E. C. (2011). Why do RNA viruses recombine? Nat. Rev. Microbiol. 9, 617-626.

Subba-Reddy, C. V., Yunus, M. A., Goodfellow, I. G., and Kao, C. C. (2012). Norovirus RNA synthesis is modulated by an interaction between the viral RNA-dependent RNA polymerase and the major capsid protein, VP1. J. Virol. 86, 10138-10149.

Sun, Q., Choi, G. H., and Nuss, D. L. (2009). A single Argonaute gene is required for induction of RNA silencing antiviral defense and promotes viral RNA recombination.
Proc. Natl. Acad. Sci. U.S.A. 106, 17927-17932.

Sztuba-Solinska, J., Fanning, S. W., Horn, J. R., and Bujarski, J. J. (2012). Mutations in the coat protein-binding cis-acting RNA motifs debilitate RNA recombination of Brome mosaic virus. Virus Res. 170, 138-149.

Sztuba-Solinska, J., Urbanowicz, A., Figlerowicz, M., and Bujarski, J. J. (2011). RNA-RNA recombination in plant virus replication and evolution. Annu. Rev. Phytopathol. 49, 415-443.

Takeshita, D., and Tomita, K. (2010). Assembly of Q\{beta\} viral RNA polymerase with host translational elongation factors EF-Tu and -Ts. Proc. Natl. Acad. Sci. U.S.A. 107, 15733-15738.

Tanne, E., and Sela, I. (2005). Occurrence of a DNA sequence of a non-retro RNA virus in a host plant genome and its expression: evidence for recombination between viral and host RNAs. Virology 332, 614-622.

Turner, P. E. (2003). Searching for the advantages of virus sex. Orig. Life Evol. Biosph. 33, 95-108.

Turturo, C., Friscina, A., Gaubert, S., Jacquemond, M., Thompson, J. R., and Tepfer, M. (2008). Evaluation of potential risks associated with recombination in transgenic plants expressing viral sequences. J. Gen. Virol. 89, 327-335.

Urbanowicz, A., Alejska, M., Formanowicz, P., Blazewicz, J., Figlerowicz, M., and Bujarski, J. J. (2005). Homologous crossovers among molecules of brome mosaic bromovirus RNA1 or RNA2 segments in vivo. J. Virol. 79, 5732-5742.
Valli, A., López-Moya, J. J., and García, J. A. (2007). Recombination and gene duplication in the evolutionary diversification of $\mathrm{P} 1$ proteins in the family Potyviridae. J. Gen. Virol. 88, 1016-1028.

van der Hoek, L., Back, N., Jebbink, M. F., de Ronde, A., Bakker, M., Jurriaans, S., et al. (2005). Increased multinucleoside drug resistance and decreased replicative capacity of a human immunodeficiency virus type 1 variant with an 8 -amino-Acid insert in the reverse transcriptase. J. Virol. 79, 3536-3543.

Visser, J. C., and Bellstedt, D. U. (2009) An assessment of molecular variability and recombination patterns in South African isolates of potato virus Y. Arch. Virol. 154, 1891-1900.

White, K. A., and Morris, T. J. (1995). RNA determinants of junction site selection in RNA virus recombinants and defective interfering RNAs. RNA 1, 1029-1040.

Wierzchoslawski, R., Dzianott, A., and Bujarski, J. (2004). Dissecting the requirement for subgenomic promoter sequences by RNA recombination of brome mosaic virus in vivo: evidence for functional separation of transcription and recombination. J. Virol. 78, 8552-8564.

Woo, P. C., Lau, S. K., Huang, Y., and Yuen, K. Y. (2009). Coronavirus diversity, phylogeny and interspecies jumping. Exp. Biol. Med. (Maywood) 234, 1117-1127.

Yamasaki, S., Sakai, J., Fuji, S. Kamisoyama, S., Emoto, K., Ohshima, K., et al. (2010) Comparisons among isolates of sweet potato feathery mottle virus using complete genomic RNA sequences. Arch. Virol. 155, 795-800.
Zemer, R., Kitay Cohen, Y., Naftaly, T., and Klein, A. (2008). Presence of hepatitis $\mathrm{C}$ virus DNA sequences in the DNA of infected patients. Eur. J. Clin. Invest. 38, 845-848.

Zhang, C. X., Cascone, P. J., and Simon, A. E. (1991). Recombination between satellite and genomic RNAs of turnip crinkle virus. Virology 184 791-794.

Zhang, X., Sato, S., Ye, X., Dorrance, A. E., Morris, T. J., Clemente, T. E., et al. (2011). Robust RNAi-based resistance to mixed infection of three viruses in soybean plants expressing separate short hairpins from a single transgene. Phytopathology 101, 1264-1269.

Conflict of Interest Statement: The author declares that the research was conducted in the absence of any commercial or financial relationships that could be construed as a potential conflict of interest.

Received: 13 December 2012; accepted: 11 March 2013; published online: 26 March 2013.

Citation: Bujarski JJ (2013) Genetic recombination in plant-infecting messenger-sense RNA viruses: overview and research perspectives. Front. Plant Sci. 4:68. doi: 10.3389/fpls.2013.00068

This article was submitted to Frontiers in Plant-Microbe Interaction, a specialty of Frontiers in Plant Science.

Copyright (c) 2013 Bujarski. This is an open-access article distributed under the terms of the Creative Commons Attribution License, which permits use, distribution and reproduction in other forums, provided the original authors and source are credited and subject to any copyright notices concerning any thirdparty graphics etc. 